\title{
Surgical management of thoracoabdominal aortic aneurysm associated with systemic lupus erythematosus
}

\author{
John G. T. Augoustides, MD, FASE, ${ }^{a}$ Alberto Pochettino, MD, ${ }^{\mathrm{b}}$ and Jeffrey Carpenter, MD, ${ }^{\mathrm{c}}$ Philadelphia, $\mathrm{Pa}$
}

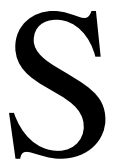

ystemic lupus erythematosus (SLE) is associated with aortitis that may cause aneurysm and/or dissection. ${ }^{1-4}$ Furthermore, the aortic syndromes associated with SLE do not typically correlate with the degree of cardiac inflammation, especially with endocarditis. ${ }^{5}$ We present a case of thoracoabdominal aneurysm (TAA) associated with Libman-Sacks endocarditis in a patient with chronic SLE. To the best of our knowledge, this is the first report of this dual presentation in a surgical patient with SLE.

\section{Clinical Summary}

A 29-year-old woman with longstanding SLE had severe back pain. Her medical history included hypertension and hemodialysis for end-stage renal disease. Her physical examination was notable for severe hypertension and an apical holosystolic murmur. She had no fever or peripheral stigmata of endocarditis. A computerized tomographic axial scan revealed an extent $\mathrm{V}$ TAA with a maximal diameter of $6 \mathrm{~cm}$ (Figure 1). She was admitted to the intensive care unit for aggressive intravenous vasodilator therapy. Myocardial perfusion imaging with thallium suggested coronary ischemia. Subsequent coronary angiography revealed no surgical disease. Transthoracic echocardiography showed normal ventricular function, mild mitral regurgitation, and a possible mitral vegetation. Subsequent transesophageal echocardiography confirmed the mitral vegetation (Figure 2), noted extensive aortic atheroma and the TAA, and excluded any further surgical valvular lesions.

The patient subsequently had multiple negative blood cultures and continued to be afebrile. The mitral vegetation was considered atypical for infectious endocarditis. After consultation with infectious disease colleagues, the clinical diagnosis of Libman-Sacks endocarditis was reached. No further management for the mitral valve was deemed necessary.

Subsequently, the patient was transferred to the operating room for surgical repair of the TAA under balanced general endotracheal anesthesia. The patient had a lumbar subarachnoid catheter placed for perioperative cerebrospinal fluid drainage. The surgical findings

From the Cardiothoracic Section, Anesthesiology and Critical Care, ${ }^{\mathrm{a}}$ the Division of Vascular Surgery, ${ }^{b}$ and the Division of Cardiothoracic Surgery, ${ }^{\mathrm{c}}$ Department of Surgery, University of Pennsylvania School of Medicine, Philadelphia, Pa.

Received for publication Aug 8, 2007; accepted for publication Aug 14, 2007.

Address for reprints: John G. T. Augoustides, MD, FASE, Assistant Professor, Cardiothoracic Section, Anesthesiology and Critical Care, Dulles 680, HUP, 3400 Spruce St, Philadelphia, PA (E-mail: yiandoc@hotmail.com).

J Thorac Cardiovasc Surg 2008;136:215-6

$0022-5223 / \$ 34.00$

Copyright (C) 2008 by The American Association for Thoracic Surgery doi:10.1016/j.jtcvs.2007.08.083

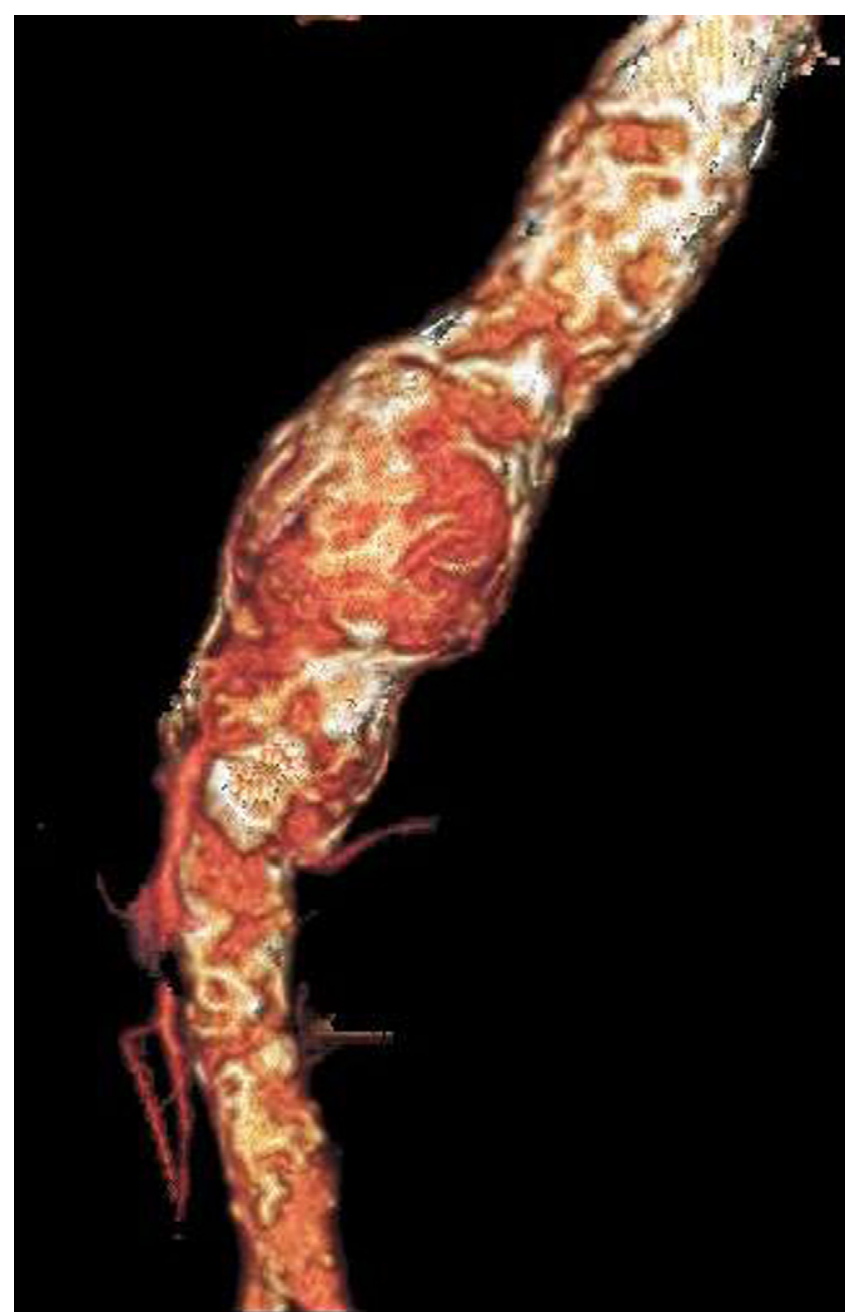

Figure 1. Three-dimensional reconstruction of the distal descending thoracic aorta and the abdominal aorta from the computed tomographic aortic study. An extent type V thoracoabdomainal aneurysm is evident.

included an extent V TAA, and diffuse eggshell aortic calcification. The patient was heparinized and supported by partial cardiopulmonary bypass (right atrial-distal abdominal aortic cannulation). The subsequent aortic repair with a $24-\mathrm{mm}$ graft was uncomplicated.

The postoperative recovery included regular dialysis, intermittent cerebrospinal fluid drainage for 48 hours, and epidural analgesia. The patient was transferred to the surgical ward on the sixth postoperative day. On the 10th postoperative day, severe hypotension and paraplegia suddenly developed. Despite immediate 


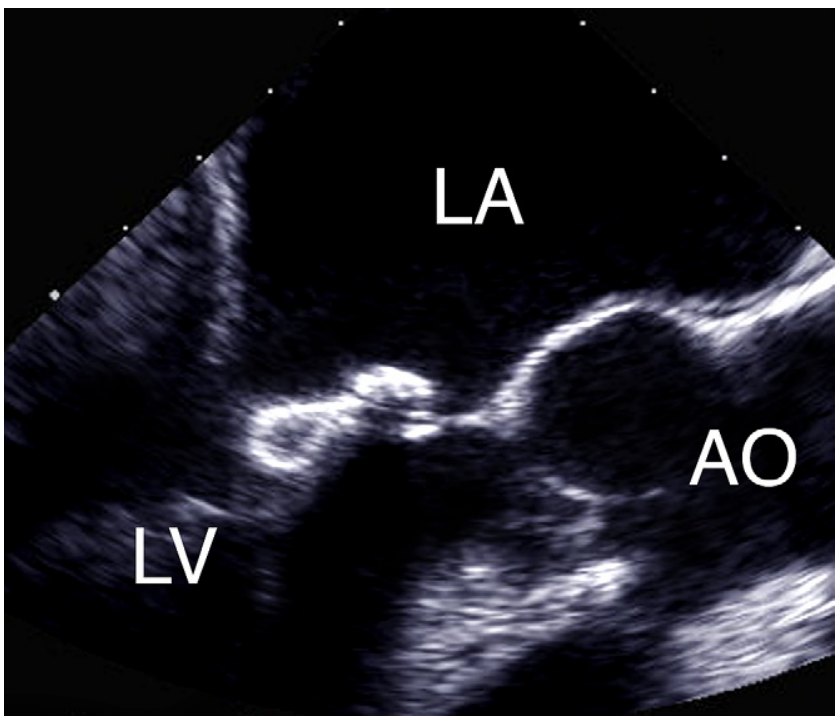

Figure 2. Transesophageal echocardiographic view showing the midesophageal long-axis view of the aortic valve with the opening of the mitral valve in diastole. The anterior mitral leaflet is calcified and has a vegetation attached to its atrial surface. The vegetation is calcified and is seen prolapsing into the left ventricle during diastole. $A O$, Aorta; $L V$, left ventricle; $L A$, left atrium.

resuscitation, the patient died of refractory ventricular fibrillation. At the request of the family, a postmortem examination was not performed.

\section{Discussion}

This patient had extensive cardiovascular disease associated with longstanding SLE: mitral endocarditis, diffuse aortic atheroma, extensive aortic calcification, and an extent V TAA. Each of these pathologic conditions significantly affected clinical management, as will be discussed herein.

Valvular disease is common in SLE, with up to $50 \%$ of patients displaying abnormalities on echocardiographic examination. ${ }^{5}$ After valvular thickening, vegetations are the second most common manifestation with a reported incidence of up to $40 \%$. Echocardiography is often unable to discern the precise pathologic status of the vegetation, such as active valvulitis, healed valvulitis, and/or thrombus. The valvulits is often ongoing and intermittient, explaining the dynamic nature of noninfectious vegetations seen in association with SLE. ${ }^{5}$ In our patient, surgery was delayed until the possibility of infective endocarditis was excluded on the basis of the absence of typical clinical features and negative blood cultures. Furthermore, left heart manipulation was avoided intraoperatively to avoid embolism from the mitral vegetation.

The surgical repair of the TAA was uncomplicated, but unfortunately the patient died before hospital discharge. The cause of her acute profound hemodynamic collapse is unclear, given the absence of a postmortem examination. A differential diagnosis related to her clinical presentation would include aortic rupture with possible dehiscence and coronary embolism from the mitral vegetation. Aortic aneurysms in association with SLE have a higher risk of anastomotic dehiscence owing to poor tissue quality, as was noted at surgery in our patient. ${ }^{1-4}$ Coronary embolism of a noninfectious mitral vegetation is in keeping with the literature. ${ }^{5}$

In summary, the clinical observation from this case is that the cardiovascular manifestations of SLE may be multiple and simultaneous. Thorough preoperative investigation will characterize and stage the lesions, allowing a rational approach to surgical therapy. The postoperative period may still represent a period of high risk beyond the typical considerations for thoracic aortic procedures.

\section{References}

1. Takagi H, Mori Y, Iwata H, Kimura M, Itokazu M, Shimokawa K, et al. Nondissecting aneurysm of the thoracic aorta with arteritis in systemic lupus erythematosus. J Vasc Surg. 2002;35:801-4.

2. Ohara N, Miyata T, Kurata A, Oshiro H, Sato O, Shigematsu H. Ten years' experience of aortic aneurysm associated with systemic lupus erythematosus. Eur J Vasc Endovasc Surg. 2000;19: 288-93.

3. Aoyagi S, Akashio H, Otsuka H, Sakashita H, Okazaki T, Tayama K. Acute type A aortic dissection in a patient with systemic lupus erythematosus. Jpn Heart J. 2002;43:567-71.

4. Washiyama N, Kazui T, Takinami M, Yamashita K, Terada H, Muhammad BAH, et al. Surgical treatment of recurrent abdominal aortic aneurysm in a patient with systemic lupus eruthematosus. J Vasc Surg. 2000;32:209-12.

5. Roldan CA, Shively BK, Crawford M. An echocardiographic study of valvular heart disease associated with systemic lupus erythematosus. N Engl J Med. 1996;335:1424-30. 